\title{
ADAPTACIÓN ACADÉMICA DE ESTUDIANTES MIGRANTES EN CONTEXTO DE FRONTERA ${ }^{1}$
}

\author{
Raúl Bustos ${ }^{2}$ \\ Joaquín Gairín ${ }^{3}$
}

\begin{abstract}
RESUMEN
El objetivo de la presente investigación es analizar las características asumidas por el proceso de adaptación académica de los estudiantes migrantes incorporados a la educación básica y media de la ciudad de Arica, Chile, contexto de investigación caracterizado por su situación bifronteriza. Se optó por un enfoque cualitativo, para apreciar la realidad a partir de los significados entregados por los sujetos que la construyen. La recolección de información combinó dos grupos focales y 15 entrevistas en profundidad. En los primeros participaron profesores que trabajan en establecimientos con presencia de estudiantes migrantes y apoderados de estudiantes migrantes de centros educativos de la ciudad de Arica. Las entrevistas se aplicaron a jefes de unidades técnico pedagógicas, y a estudiantes migrantes, desarrollándose bajo la modalidad del relato autobiográfico. El proceso de codificación fue abierto y se empleó el análisis de contenido, a partir de cadenas textuales. Los resultados señalan que el contexto en estudio cuenta con una limitada predisposición a la interculturalidad. Las escuelas parten de la premisa de que es el estudiante quien debe adaptarse al sistema escolar, considerando como tarea la realización de procedimientos normalizadores. Este hecho se justifica por los buenos rendimientos obtenidos por este grupo de estudiantes.
\end{abstract}

Palabras clave: educación, frontera, migración.

\section{STUDENT ACADEMIC ADAPTATION OF MIGRANTS IN BORDER CONTEXTS}

\section{ABSTRACT}

The objective of this study is to analyze the academic adaptation process experienced by migrant students in elementary and secondary schools in the city of Arica, a research context characterized by its close proximity to an international border. This study takes on a qualitative approach to appreciate reality as it is constructed from perspective of migrant students. The data collected includes two focus groups and fifteen in-depth interviews. The focus groups gathered input from teachers who work in educational establishments with migrant students and parents

Artículo resultante del proyecto de investigación "Perfil académico y sociodemográfico de los estudiantes migrantes de la Universidad de Tarapacá", Proyecto Interno de Investigación en Educación, Universidad de Tarapacá, código 5718-16.

2 Departamento de Educación, Universidad de Tarapacá, Arica, Chile. Contacto: rbgonzalez@ academicos.uta.cl

3 Departamento de Pedagogía Aplicada, Universidad Autónoma de Barcelona, Barcelona, España. Contacto: joaquin.gairin@uab.cat 
of migrant students that attend educational establishments in the city of Arica. The interviews were conducted on school leaders as well as migrant students and in the latter case, maintained an autobiographical focus. An open coding technique was applied to analyze content based on strings of text. The results indicate that the context studied demonstrates limited predisposition to accept interculturality. The schools are based on the premise that it is the student who must adapt to the school system and take on the task of standardizing their own behavior. This fact is justified by the positive learning outcomes obtained by the group of students that are able to do so.

Keywords: border, education, migration.

\section{Introducción}

Las características de una realidad globalizada y abierta a los intercambios constantes chocan, cada vez más, con los mecanismos de control del orden social que las comunidades contemporáneas están llevando a cabo. El desarrollo de muros entre fronteras (Estados Unidos con México, Israel con los países vecinos, España con Marruecos, etc.); los límites de la apertura de fronteras a los desplazados por guerras en Europa y Asia o la marginación que sufren los inmigrantes en muchos países son una evidencia de lo que señalamos. En consecuencia, no es extraño que la globalización se interprete como un proyecto ideológico que daría lugar a formas extremas de exclusión de gran parte de la población mundial (Lube, Penna, Vicuña y Pérez, 2015).

Stefoni (2003) indica que las tasas negativas de crecimiento demográfico y los niveles de desarrollo alcanzados por algunas economías generan una necesidad de mano de obra para cubrir las tareas poco atractivas para los nacionales ${ }^{4}$. De hecho, las migraciones movilizan principalmente gente en edad económicamente activa ${ }^{5}$ y muchas veces impulsan cargas nacionalistas que justifican la explotación del migrante (Pizarro, 2011).

4 Alvites y Jiménez (2011) señalan que: "La tasa de recambio en Chile es 1,7\%, mientras que la de Perú es de 2,6\%. Un estudio de la Universidad de Chile muestra que el 7,8\% de los partos atendidos en 2010 fue de madres extranjeras" (Alvites y Jiménez, 2011, p. 122).

5 El informe sobre migraciones del año 2013, elaborado por la Oficina Internacional para las Migraciones, OIM, señala que el total del contingente de migrantes internacionales al 2010 era de 214.199 .000 personas. Un $15,1 \%$ de estos migrantes tendría entre 0 y 19 años; un $73,8 \%$ estaría entre los 20 y 64 años, y un 11\% tendría 65 años o más. 
Los fenómenos migratorios asumen así características estructurales, que han potenciado que la institucionalidad reaccione de manera inoportuna, por desconocimiento o por la presencia de estereotipos poco beneficiosos para una real inclusión de migrantes, muchos de ellos niños y jóvenes en edad escolar (Essomba, 2006).

Explorar las estrategias de éxito académico empleadas para lograr la plena inclusión de los estudiantes migrantes (objetivo prioritario en sistemas educativos progresistas) se transforma, en estas circunstancias, en una empresa de ruptura de las relaciones asimétricas emergidas en el contexto descrito. Al respecto, se plantea analizar la situación académica de los estudiantes migrantes en los establecimientos de educación básica y media de la ciudad de Arica (Chile), y analizar las estrategias educativas que se utilizan para dar respuesta a las problemáticas identificadas en esta zona de frontera.

Se entiende como estrategia el conjunto de actuaciones y procesos que configuran procedimientos considerados adecuados para el logro de la adaptación académica de los migrantes. Se distinguen entre estrategias de aula y estrategias institucionales:

a. Estrategias de aula, que pueden ser de tres tipos (Lanza y Sánchez, 2013):

- Estrategias de apoyo: abarcan la motivación, las actitudes y el afecto, buscando sensibilizar al estudiante hacia el aprendizaje.

- Estrategias cognitivas: se asocian a la integración del nuevo aprendizaje con el conocimiento previo. Las más importantes son repetición, selección y elaboración.

- Estrategias metacognitivas: son las que ayudan al estudiante a conocer lo que sabe acerca de la tarea, su dificultad y cómo resolverla.

b. Estrategias institucionales: se trata de aquellas puestas en funcionamiento por la organización y que se asocian con el "apoyo social" (Taylor et al., 2004, p. 355, citado por Hombrados y Castro, 2013). Estos últimos particularizan tres dimensiones de apoyo social: emocional, instrumental e informacional. El primero 
representa el sentimiento personal de ser parte de una red; el apoyo instrumental hace referencia a la posibilidad de poder disponer de ayuda directa; por último el apoyo informacional consiste en la provisión de consejo o guía para ayudar a las personas a resolver sus problemas (Hombrados y Castro, 2013).

En este contexto, el objetivo del estudio es analizar las estrategias de adaptación académica que se vinculan a los migrantes en la ciudad de Arica, Chile, en pleno contexto de frontera.

\section{El marco contextual}

La Región de Arica y Parinacota es la puerta norte de Chile, lugar

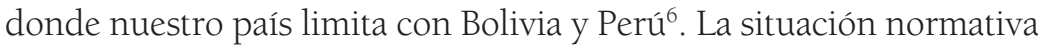
es uno de los elementos que otorga particularidad al fenómeno migratorio en esta zona, ya que permite el libre tráfico entre Tacna (Perú) y Arica (Chile) para los ciudadanos de ambos países, con la sola presentación del documento de identidad, siempre y cuando el desplazamiento cumpla con la condición de la permanencia de siete días en el territorio de destino.

En cuanto al tema en estudio, la Constitución de la República de Chile garantiza el acceso a la educación de todos los niños, niñas y jóvenes que residen en el país y, además, algunos de los principios inspiradores de la Ley n 20.370 (Ley General de Educación) son la integración e interculturalidad. No obstante, el acceso a la educación, aunque importante, es insuficiente (Alvites y Jiménez, 2011) ${ }^{7}$.

6 De acuerdo con los antecedentes de la Policía de Investigaciones de Chile, desde los años 2001 al 2008, la región casi ha duplicado su movimiento migratorio, pasando de 2.614.653 a 4.640658 personas. El número de ciudadanos inmigrantes es de 12.299 en esta región, de los cuales 5.996 personas provienen de Bolivia y 4.821 de Perú. El número total de personas inmigrantes conforman un 5,8\% de la población total de la región (Instituto Nacional de Estadísticas, INE, 2012).

7 En la región de estudio, la participación de los estudiantes extranjeros en relación con el total de estudiantes matriculados, para el año 2013, alcanzó el 2\% (Centro de Estudios Mineduc, 2013). De acuerdo con Vicuña y Rojas (2015), el 60\% de los estudiantes migrantes estudia en zonas urbanas de la región, y un $40 \%$ en rurales. Asimismo, el $75 \%$ se sitúa en el sector municipal. La mayor cantidad de estudiantes extranjeros corresponde a las nacionalidades peruana y boliviana, lo que equivale a un $91 \%$ del total de estudiantes extranjeros de la región. 
El problema no solo es legal, sino que también existe la tendencia a infravalorar la cultura del migrante (Carrasco, Pàmies y Bertrán, 2009), basándose en prejuicios etnocéntricos que justifican la discriminación (Vera, 2009). Particularmente, la relación con los estudiantes provenientes de Perú y Bolivia está "todavía centrada en una mirada de exaltación belicista y menoscabo de la identidad de los niños y sus familias peruanas y bolivianas" (Alvites y Jiménez, 2011, p. 123). Estos mismos autores citan un informe elaborado para Santiago de Chile, que mostró que de 200 niños y niñas inmigrantes, el 32\% de sus padres manifestó haber sufrido discriminación grave en la escuela, incluso por parte de una profesora jefe y hasta de un director de colegio. Otro estudio del mismo año, citado por los mismos autores, señaló que:

El 46\% de niños y adolescentes entrevistados consideraron que una o más nacionalidades son inferiores a la chilena; Perú y Bolivia, países especialmente menoscabados en los programas de historia actuales, y de donde proviene la mayoría de los alumnos inmigrantes, concentran más de la mitad de esta discriminación (Alvites y Jiménez, 2011, p. 123).

Así mismo, dicho estudio señala que en los colegios con presencia de alumnos extranjeros, el prejuicio de superioridad hacia otras nacionalidades disminuyó de $46 \%$ a 31\%, lo que evidencia las oportunidades de la educación inclusiva (Alvites y Jiménez, 2011)

Sin embargo, la experiencia de exclusión genera respuestas en el estudiante que pueden crear desconfianzas y deslegitimización de la autoridad como parte de una "identidad del excluido" que se consolida, a la vez que le resta valor, "desmarcándose" de los estigmas de éxito dominante (Figueroa, 2015).

Cabe señalar que, en Chile, el rendimiento académico de los estudiantes extranjeros no difiere del de los estudiantes nacionales,

8 La Organización de las Naciones Unidas destaca que la población migrante internacional en Chile ha crecido de 107.501 en 1990 a 398.251 en el 2013. Los grupos migrantes más numerosos en Chile son la colonia peruana $(53,2 \%)$, la argentina $(23,8 \%)$, la boliviana $(9,6 \%)$, la ecuatoriana $(7,6 \%)$ y la colombiana $(5,5 \%)$. 
incluso lo supera en la enseñanza técnico-profesional industrial de niños y de adultos (Centro de Estudios Mineduc, 2013).

Para el caso de la región en estudio, los resultados generales obtenidos por sus establecimientos en las pruebas de medición de calidad (Sistema Nacional de Evaluación de Resultados de Aprendizaje, Simce), comparados con los conseguidos por su grupo socioeconómico de referencia a nivel nacional, son negativos ${ }^{9}$. Además, si consideramos los datos de la Prueba de Selección Universitaria (PSU), se puede apreciar que la Región de Arica y Parinacota está muy por debajo de los promedios nacionales en la misma modalidad ${ }^{10}$.

En este contexto, y de acuerdo con los datos proporcionados por el Ministerio de Educación de Chile (Mineduc), el rendimiento académico de los estudiantes migrantes en Arica y Parinacota si bien mantiene la tendencia mostrada en el resto del país, sí presenta diferencias en el rendimiento que es favorable a los estudiantes migrantes. Esto es especialmente notorio en la enseñanza media técnico profesional comercial de niños y jóvenes, donde el rendimiento de extranjeros y nacionalizados supera bastante al de los estudiantes nacionales, a diferencia de la situación nacional donde el rendimiento de los tres grupos es equivalente. El rendimiento superior de los estudiantes extranjeros también se aprecia en el caso de la enseñanza media técnico profesional industrial de niños y jóvenes y en la enseñanza media técnico profesional técnica. Por su parte, en la enseñanza media técnico profesional agrícola de niños de la Región de Arica y Parinacota un 5\% de su matrícula está compuesta por estudiantes migrantes. El rendimiento de los estudiantes extranjeros para esta especialidad es marcadamente superior, y sobresale al que obtienen los estudiantes migrantes a nivel nacional, el cual es similar al de los nacidos en Chile.

Cuando se consulta a los estudiantes migrantes acerca de las asignaturas que les presentan mayor facilidad y las que aparecen 
más complicadas, llama la atención que aquellas consideradas generalmente más complejas, les resultan fáciles, particularmente Matemática, Química y Filosofía. Al mismo tiempo, es llamativo que las asignaturas declaradas más complejas para este grupo vulnerable sean las que, aparentemente, son consideradas como aquellas que tienden a mejorar los promedios de los estudiantes nativos por su menor dificultad (Artes, Música y Tecnología). Pese a dicha percepción, las estadísticas de rendimiento de los estudiantes migrantes señalan que estas últimas son aquellas en las que presentan mejores calificaciones.

\section{Marco teórico: gestión social de la diversidad y educación}

La sociedad occidental intenta tradicionalmente la generación del hombre útil y uniforme en sus comportamientos, marginando la diferencia (Sauquillo, 1987). Esta estructura denominada "sociedad normalizada" y la integración a ella, se relaciona con el tránsito de los individuos excluidos a la estructura de la sociedad dominante, lo que se expresaría en políticas sociales, de vivienda, educación o salud (Morales y Molina Prieto, 2003).

Cuando la sociedad normalizada promueve la integración, obvia las carencias personales que serían obstáculos al logro de una mejor calidad de vida. De esta manera, la exclusión de ciertos grupos tiende a ser invisible al catalogarse como "normal" (Sánchez y Gil, 2015).

En los últimos años, el término "inclusión" ha sustituido al de "integración". La idea de integración parte del supuesto de que es necesario "integrar" al alumno "diferente". Esta interpretación conlleva la imposición de los patrones vitales de los grupos dominantes (Dovigo, 2014).

Según Booth y Ainscow (2002), desde la perspectiva educativa, "la inclusión se concibe como un conjunto de procesos orientados a eliminar o minimizar las barreras que limitan el aprendizaje y la participación de todo el alumnado" (p. 9). Hablamos así de una educación estándar que, con ayudas, permite la incorporación de 
los "diferentes" frente a la propuesta de una educación que se adapta y proporciona las respuestas que cada persona y colectivo social necesita.

Pàmies (2011) y Poveda (2003) señalan los riesgos que trae para los estudiantes migrantes la participación en procesos donde sus referentes culturales son ignorados. Esta realidad se acentúa al reconocer la actitud general del profesorado, de desinterés por conocer el contexto en la que desempeña su labor y la creencia de que la situación se "normalizará con el tiempo" (Abajo y Carrasco, 2004).

Gairín (2014) remarca que cualquier intervención institucional que promueva el cambio educativo debe enfrentarse a un "cambio global" que incluya desarrollo curricular, organizativo, profesional y comunitario. Por su parte, Gómez (2005) divide las estrategias de adaptación académica en dos: medidas de carácter ordinario y medidas de carácter extraordinario, asumiendo como una tarea propia de la labor docente la planificación para un aula diversa. Entre las medidas de carácter extraordinario, el autor señala las que deben ejecutarse en virtud de grupos que, con las medidas ordinarias, no estarían en condiciones de alcanzar los mínimos exigidos.

En América Latina, y a pesar de que en los últimos años se ha puesto en marcha una serie de medidas gubernamentales alineadas con la interculturalidad, dichas iniciativas no han cumplido con sus expectativas iniciales, principalmente porque se han particularizado en contextos indígenas y se han implementado como medidas de reparación social, dejando fuera de las mismas a colectivos emergentes de migraciones contemporáneas (Mondaca y Gajardo, 2013).

No obstante, en los últimos años se ha multiplicado el número de investigaciones en el tema de inclusión educativa (Figueroa, 2015, Jiménez y Fardella, 2015; Matus y Rojas, 2015) y su situación en contextos interculturales (Bustos y Gairín, 2017; Joiko y Vásquez, 2016; Marín, 2014; Riedemann y Stefoni, 2016), lo que señala la contemporaneidad de esta problemática. Sin embargo, es necesario reconocer que lo escrito en planes, decretos y proyectos educativos no siempre se lleva a la práctica en la escuela, siendo aún muchas las 
dificultades que enfrentan los estudiantes migrantes para su inclusión en las sociedades receptoras (Poblete, 2006).

\section{Marco aplicado. Diseño y justificación metodológica}

De acuerdo con el objeto de estudio y los objetivos planteados, se optó por un enfoque etnográfico, considerando que solo se puede apreciar la realidad de análisis como una construcción posible de conocer a partir de los significados entregados por los sujetos que la elaboran. Sin embargo, es necesario subrayar que en educación, lo que se hace es una adaptación de la etnografía, ya que el fenómeno de interés es el proceso educativo. De manera concreta, la investigación expuesta se reconoce como "microetnografía", que:

No es por tanto el tiempo en el campo lo que define una microetnografía, sino la mirada y la escucha del investigador, el foco de la observación y la manera de construir el relato etnográfico para que refleje la complejidad de los contextos humanos (Hernández, Martínez y Montané, 2014, p. 67).

La noción de microetnografía, entonces, tiene dos componentes: un abordaje puntual e intensivo, y el estudio de "grandes" temas a través del análisis de "pequeños" momentos de actividad humana.

Como técnicas de recolección de información se utilizaron la entrevista en profundidad y los grupos focales, para poder lograr una doble aproximación: la del investigador y la de los participantes (Jackson, 2001; Poveda, 2003). Concretamente, se realizaron dos grupos focales, con 14 y 12 participantes respectivamente, compuestos por profesores que trabajaban en establecimientos con presencia de estudiantes migrantes y apoderados de estudiantes migrantes de más de 12 centros educativos de la población de Arica ${ }^{11}$. El número de participantes de cada grupo focal obedeció a una doble

11 De los 14 profesores, la mitad se desempeña en educación básica y el resto en educación media. Asimismo, los centros tenían la siguiente distribución: nueve con dependencia municipal y cinco particulares subvencionados. En cuanto a los apoderados, de los 12 consultados, seis eran peruanos, cuatro bolivianos, un colombiano y un paraguayo. A su vez, cinco de estos apoderados tenían hijos en educación básica y siete en educación media. Finalmente, cuatro estudiaban en centros municipales y ocho en colegios particulares subvencionados. 
estrategia de convocatoria, la que considerando la normal deserción a estas actividades por parte de los convocados, incluyó la invitación a profesores y apoderados de estudiantes de Pedagogía que realizaban su práctica en los establecimientos seleccionados, en conjunto con la metodología de "bola de nieve", lo que incluyó a referenciados de los propios convocados. Además, en el caso de los apoderados migrantes, se contó con el patrocinio de la ONG Ciudadano Global, entidad confiable para los apoderados a quienes se dirigía la convocatoria.

Posteriormente, se entrevistó en profundidad, y bajo la técnica del relato autobiográfico, a 15 estudiantes migrantes, optándose por aquellos mayores de 12 años, ante el temor de que los estudiantes menores no tuvieran experiencias significativas para el estudio, o bien, no las pudieran relatar con detalle ${ }^{12}$. En las entrevistas se desarrolló una aproximación mediante relatos de vida cruzados contextualizados o "microcampos" (Moscoso, 2014).

La diversidad de fuentes y la subordinación de las cuestiones planteadas para los objetivos del estudio permitieron la triangulación de informantes: estudiantes migrantes, profesores y apoderados de alumnos migrantes. Los datos obtenidos en el estudio de campo, realizado durante el segundo semestre del año 2015 y primer trimestre del año 2016, se categorizaron atendiendo a criterios temáticos y, posteriormente, se codificaron. El proceso de codificación fue abierto, tomando las categorías existentes implícitamente en las preguntas que guiaron la entrevista como categorías iniciales y formulando otras cuando este repertorio se mostró ineficaz. Posteriormente, se empleó el análisis de contenido, para profundizar sistemáticamente en la información obtenida y clasificarla. El análisis de expresiones orales se realizó a partir de las transcripciones escritas de las mismas.

Finalmente, el establecimiento de relaciones entre categorías permitió discriminar la información relevante para el objeto de estudio. Este proceso se realizó a partir de cadenas textuales,

12 Los estudiantes migrantes entrevistados, cursaban estudios en colegios municipales y particulares subvencionados en la ciudad de Arica, nueve de ellos en educación media y el resto en educación básica. Siete eran peruanos, cuatro colombianos, tres bolivianos y un panameño. 
identificadas y delimitadas por una frase principal del texto, para luego analizar por separado dicha cadena y comprobar si entregaba información de un solo tema o de varios (Gil, 1994). En términos del análisis de los datos, se optó por el uso de matrices de doble entrada donde se alojó la información verbal en relación con los objetivos del estudio (Miles \& Huberman, 1994).

En síntesis, se prefirió realizar un análisis que rescata los aportes de la teoría fundamentada (Glaser $\&$ Strauss, 1967; Pidgeon \& Henwood, 1997) y el aporte de investigadores como Vásquez y Martínez (1996), quienes sugieren no forzar los datos y partir de aquellos que entrega el propio trabajo de campo.

\section{Resultados del análisis de grupos focales y entrevistas $^{13}$}

En el presente apartado se exponen los resultados obtenidos del análisis de las transcripciones de los grupos focales y entrevistas a informantes clave. La información se presentará describiendo tres dimensiones relacionadas causalmente, a la luz de lo expresado por los participantes en la investigación: elementos de contexto, rendimiento académico y estrategias de adaptación académica. La relación entre estas dimensiones se representa por las fuentes a partir del contexto particular que Arica presenta al ser zona de frontera y políticamente compartida por Chile y Perú a lo largo de su historia, promoviendo el habitual contacto entre estudiantes transfronterizos. Este hecho incidiría en los rendimientos adecuados de los estudiantes migrantes (especialmente los provenientes de Perú), lo cual es relevante en virtud de la necesidad o no de aplicar estrategias de adaptación a partir de los virtuales problemas de rendimiento que

13 Con el fin de mantener el anonimato de las fuentes consultadas, se utilizó un sistema simple de codificación: a los profesores se les designó como "Profesor", seguido por las iniciales de la institución educativa en donde se desempeña, por ejemplo: (Profesor AC); a los jefes de unidad técnico pedagógica se les designó por la sigla "UTP" y las iniciales del establecimiento donde se desempeña, por ejemplo: (UTPFN); a los apoderados de estudiantes migrantes se les designó con la nacionalidad de origen, seguido por la edad, por ejemplo: (Peruano48); a los estudiante migrante se les designó con la palabra "Estudiante", el país de origen y la edad, por ejemplo: (Estudiante peruana, 16). Al final de cada cita se incluye el número de página del cuaderno de transcripción de la entrevista. 
pudieran presentarse, obviando cualquier otra variable que pudiera demandar dicha atención.

\section{a. Elementos de contexto}

La situación de los alumnos migrantes no es un tema de reflexión para los docentes, lo que se justifica por la aparente adaptación de dichos estudiantes al sistema educativo local: "el problema no se aborda porque no se visualiza, porque no hay quejas, denuncias ni nada de eso (Profesor de CCRSH: 6).

En los contextos rurales, se percibe la normalización y la homogeneización cultural como su principal tarea: "se les apoya en los trámites de residencia, y para que saquen el RUT chileno. (...) Lo que sí hay que tratar de imponer nuestros valores y para ello aprovechamos los actos cívicos del lunes" (UTPDS: 3). Es importante resaltar el énfasis que se hace en la imposición de la nueva cultura, asimilación que está lejos de la letra y el espíritu de los modelos inclusivos o de respeto a los orígenes y a las opciones personales.

Los profesores señalan que los principales núcleos de conflicto entre estudiantes migrantes y nacionales nacen por la transmisión de mitos a nivel familiar, y muchas de las conductas que tanto profesores como estudiantes catalogan como normales están lejos de ser deseables: "Una vez me gritaron peruano... (señala una grosería), pero era jugando a la pelota" (Estudiante peruano, 14:3).

Por otro lado, se reconoce una conducta protectora de la familia migrante, consistente en la prohibición de los contactos sociales de los niños con otros niños fuera de la escuela, lo que probablemente dificulta el proceso inclusivo: "cuando estábamos en vacaciones era triste, porque siempre estaba en mi casa, no salía" (Estudiante colombiano, 14:2). Así, los estudiantes migrantes construyen sus principales relaciones sociales en Chile con sus compañeros de curso: "Tengo amigos aquí, pero fuera no. No conozco a nadie de fuera" (Estudiante panameño, 12:4). 


\section{b. Rendimiento académico}

Los estudiantes migrantes presentan ventajas en su rendimiento académico que, según los docentes, se debe a una mejor actitud hacia el estudio y a la presencia de contenidos tratados en su experiencia escolar en el país de origen: "En general, su rendimiento es mejor al del resto de los estudiantes (...). La principal diferencia es que ellos estudian y su formación inicial es mejor" (UTPFN:1).

Las diferencias identificadas por los estudiantes migrantes entre el sistema educativo de su país de origen y el de Chile las explican cuando señalan que su experiencia escolar en el país de acogida es menos exigente, particularmente en el caso peruano. Este hecho es reconocido también como un potencial problema para su reinserción educativa, en el caso de un retorno al país de origen: "Comparado con mi país natal, mi hijo está atrasado (...). Si yo lo llevo a mi país natal a seguir el mismo curso, estaría desnivelado" (Peruano, 48:3).

En términos específicos, los jefes de unidad técnica señalan diferencias en el caso de los estudiantes migrantes provenientes de contextos rurales, particularmente bolivianos, que presentarían ciertas desventajas en su formación.

\section{c. Estrategias de adaptación académica}

En cuanto al uso de estrategias, los docentes señalan que no son necesarias, considerando la motivación intrínseca de los estudiantes migrantes: "Ellos llegaron igual con una actitud a integrarse" (Profesor de NAC:3). Los profesores perciben el uso de estrategias como el resultado de la acción del mismo estudiante migrante: "Tenemos cierto grado de diferencia hacia ellos, en la medida de que ellos se los ganen" (Profesor de LA-1OPP:3).

Las jefaturas técnicas señalan el desacuerdo de los estudiantes migrantes con el trato que los estudiantes nacionales tienen hacia los profesores: "me llama la atención que algunos alumnos no respeten mucho y los profes, de repente, tendrían que ser un poquito más estrictos con algunos niños que son asî" (Peruano, 42:4). 
Es probable que la actitud hacia los docentes por parte de los estudiantes migrantes se relacione con el temor a recibir castigos físicos: "Se les ve temerosos. Tienen miedo a los golpes. A veces me preguntan 'profesor ¿no me va a pegar?"' (UTPDS: 2). Los estudiantes destacan la relación más cercana con los profesores chilenos: "porque allá los profesores nos tratan mal y aquí no" (Estudiante boliviano, 15:5).

Las principales discrepancias entre las fuentes aparecen en torno a las razones del relativo éxito académico del grupo de estudiantes en estudio. Los docentes encuentran el origen de esta situación en las necesidades de aceptación social. Sin embargo, para los padres, se debe al menor nivel de exigencia que experimentan en Chile: "Aquí creen que a los niñitos no se les puede exigir porque se pueden estresar" (Peruano, 29:3). Es así que muchas de las actitudes y costumbres presentes en las escuelas nacionales no son consideradas beneficiosas por los padres migrantes, por lo que restringen su adopción en sus hijos: "No tienen mucho respeto a los profesores, incluso a las mamás mismas, no les respetan mucho" (Boliviano, 25:3).

Algunos padres migrantes señalan que la relativa falta de disciplina en los establecimientos educacionales se relaciona con un retroceso en el desarrollo académico de sus hijos: "Mi hijo ha empeorado, ahora es mucho más flojo. Y me dice, 'pero papá para qué estudio si no sirve"' (Peruano, 29:4).

Muchos profesores señalan que no debe haber diferencia entre sus alumnos, obviando las realidades y experiencias vitales que pudieran provocarlas. No obstante, esta "igualdad" debe ser reafirmada a través de procedimientos normalizadores, como la obtención de certificación nacional:

A fin de establecer un cierto control hay una ley que exige que ellos tengan su residencia legalizada. Entonces hay algunos casos en que el niño no tiene ningún documento en regla, pero por la convención de los Derechos del Niño, tú no le puedes negar educación. Ellos quedan con un RUT provisorio, un RUT 
100 millones, entonces se le va pidiendo al apoderado que vaya regularizando, quienes a veces se demoran más de un año (Profesor de E-15:4).

Lo interesante es reconocer que en torno a lo descrito, se evidencia la representación que los docentes tienen de lo que es una educación inclusiva, lo que revela un discurso asociado a los afanes homogeneizadores expuestos en otros apartados:

Entonces, si queremos incluir tenemos que hacer lo mismo para todos, y si a él le cuesta o se siente más avergonzado porque recién viene llegando, bueno, hay que tratar de explicarle, integrarlo más, pero no hacer, por ejemplo, actividades aparte para él, porque estamos hablando de un sistema inclusivo ahora en la educación. Necesitamos que todos tengan la misma educación, no diferenciarlo (Profesor de ED-4RI:4).

La presencia de estas estrategias es vista como un elemento de discriminación y, por ende, poco deseable. Hay algunas iniciativas coyunturales que asumen de manera incipiente cierta formalidad, tal vez sin mayor conciencia, en estrategias motivacionales e inclusivas:

Hacemos actividades específicas cuando hay actividades deportivas. Te cito el Mundial de Fútbol, ocasión que se le permite al alumno migrante que venga con la polera de su país, para que ellos recuerden que tienen que valorar su origen. Porque hay algunos que reniegan, y algunas veces puede ser muy traumático. Pero después se dan cuenta que Chile es un país tranquilo, el país que andaban buscando. Pero como te digo, se les hace valorar su origen y que el resto valore el origen de ellos, por eso lo del Mundial. (...) aunque hubo varios de ellos que usaron la camiseta de Chile, entonces se les respeta, pero ellos toman su decisión (Profesor de E-15:5).

Como puede apreciarse en la cita anterior, se reitera la imagen presente en los docentes respecto de ser Chile el "país soñado" en el que cualquier persona quisiera desarrollar su proyecto de vida. En cuanto a la existencia de estrategias de control emocional, los profesores responsabilizan de esta función a otros profesionales de 
apoyo a la docencia: "Ese tipo de estrategias a nivel institucional creo que no existe, pero es el deber de orientación velar por la integridad personal y emocional de los estudiantes, previo informe del profesor jefe" (Profesor de LA-1OPP:5).

Para los estudiantes migrantes, reconstruir las estrategias de estudio empleadas genera cierta dificultad, principalmente por desconocimiento en torno a la presencia o no de estas en sus prácticas estudiantiles. No obstante, la gran mayoría de ellos señala el uso de resúmenes y la búsqueda de videos descargables de internet. Por otro lado, la mayoría de los estudiantes migrantes también señala estudiar con otra persona, ya sea un familiar o un compañero: "Cuando tengo prueba estudio a veces en el descanso con otros compañeros, y en mi casa. Estudio con lo que me enseñó el profesor y yo tomé nota. Escribo resúmenes, aunque viendo videos aprendo mejor" (Estudiante colombiana, 14:5).

Preparar un ambiente de estudio con música, y la elaboración de resúmenes son elementos repetidamente mencionados por los estudiantes como características fundamentales de su contexto de estudio. Por otro lado, en la mayoría de los casos, la memorización es la estrategia cognitiva más empleada por los estudiantes. Solo el estudio de las matemáticas prescinde de la memorización: "Estudio memorizando las cosas. Estudio solo, con el cuaderno o el portátil. Siempre memorizo las cosas y si son de cálculos, practico algunos ejemplos" (Estudiante boliviano, 15:5).

En cuanto a las estrategias de planificación para el estudio, se puede desprender que esta actividad no constituye una prioridad. Mayormente la planificación se traduce en una organización cronológica de actividades, o incluso solo un calendario de evaluaciones: "Sí, los domingos. Cuando ya es fin de semana, me pongo a estudiar un poco. El sábado juego un poquito y luego estudio un poco, luego vuelvo a jugar el resto del día y el domingo es el día en que más estudio" (Estudiante panameño, 12:5). Muchas veces el buen rendimiento de los estudiantes migrantes, o las facilidades encontradas en el sistema educativo nacional, ha llevado a que los estudiantes no consideren necesario planificar. 
En cuanto a las diferencias vinculadas directamente con las maneras y calidad de los aprendizajes que los estudiantes migrantes perciben entre su país de origen y el de acogida, las principales apreciaciones abordan la ausencia de aprendizajes entre los estudiantes nativos, y los que ellos ya poseen y la diferente disposición al estudio, aunque no lo adjudiquen a la diferencia de origen: "Me llamó la atención cuando llegué, de que muchos compañeros no entendían lo que explicaban porque carecían de la base, había niños que no se sabían las tablas de multiplicar, y me sorprendió eso. En eso había diferencia, no había mucho interés, me daba cuenta que carecían de cosas básicas" (Estudiante peruano, 16:7).

\section{Discusión}

El carácter fronterizo de la Región de Arica y Parinacota otorga particularidad al fenómeno descrito, especialmente porque la cotidianidad de los movimientos de población, y más aún, la raigambre histórica de los contactos entre peruanos, chilenos y bolivianos en la región, desbordan los esquemas analíticos emanados desde la metrópoli y tensionan conceptos anteriormente considerados como incuestionables, tales como nación, identidad y exclusión (Lube et al., 2015). Entre dichas particularidades podemos mencionar la invisibilidad del migrante peruano y boliviano, que genera una distinción con "el nuevo migrante" venido de Colombia, Ecuador o Centroamérica.

Como ya se ha establecido, la idea de orden social en el contexto de estudio presupone un grado de homogeneidad interna. Al respecto, la existencia de estereotipos y generalidades puede dar cuenta de la persistencia implícita de la idea de "raza", reinterpretada sobre la base de estigmas de alteridad como el acento, los modales, la religión, entre otros (Balibar \& Wallerstein, 1998). Este "racismo de entrada" (Zanetti, 2015) subyace en la tradicional percepción de incapacidad parental del migrante, o en las diferencias percibidas por este en relación con el nacional, o incluso entre ellos, dependiendo del país de origen. 
Por otro lado, la opinión común de los docentes, en cuanto a la no necesidad de desarrollar estrategias de inclusión, porque a todos se les trata igual, se identifica con el racismo de "salida" (Zanetti, 2015) donde la inactividad mantiene las diferencias, negando su importancia.

Por otro lado, los resultados de la investigación tienden a mostrar, al igual que lo señalado por Joiko y Vásquez (2016), que el paradigma desde el que se interpreta la relación con el "otro" es el de integración por sobre el ideal de inclusión propuesto desde la política pública.

Convencionalmente, en el contexto de estudio, el primer indicador evidente que se hace presente al momento de evaluar el proceso de adaptación académica del estudiante migrante se refleja en las calificaciones e informes de conducta, los que no necesariamente podrían mostrar la profundidad misma del proceso. Por el contrario, la argumentación que señala que las iniciativas de inclusión para estudiantes migrantes son innecesarias debido a sus rendimientos obtenidos, se acerca a una de las estrategias de negación del racismo, planteadas por Riedemann y Stefoni (2015) y de manera sutil informa del rol otorgado a la evaluación, como herramienta normalizadora, de acuerdo con el discurso de los docentes.

Abundante literatura reseñada en el presente trabajo tiende a asociar las estrategias de apoyo a colectivos vulnerables con tareas de "apoyo social" (Taylor et al., 2004 citado por Hombrados y Castro, 2013). Este concepto podría dar cuenta de elementos que no necesariamente tendrían su reflejo en el rendimiento, aunque probablemente sí incidirían en actitudes evaluadas disciplinariamente.

En relación con lo anterior, los informantes entrevistados, tal como señalan Hombrados y Castro (2013), varían en su evaluación de apoyo social, dependiendo de quién entregue dicho apoyo, pues se adjudican distintos roles a cada participante del proceso educativo. Es así que la gran mayoría de los estudiantes migrantes destaca el apoyo emocional de los docentes, traducido en una mayor cercanía respecto de la experimentada con sus profesores en su sociedad de origen. Por 
otro lado, los apoderados de estudiantes migrantes subrayan el apoyo informacional que se manifiesta a través de reuniones y entrevistas. Finalmente, desde los establecimientos educativos como institución se acentúa el apoyo instrumental, traducido en la entrega de materiales de estudio, alimentación y uniformes, lo que aparece coherente con los caminos de integración operados desde la institucionalidad.

De esta manera, la sociedad chilena, como expresión de la sociedad normalizada, responde a los anhelos de las minorías migrantes con una actitud caritativa (Sánchez y Gil, 2015). Se reproduce el esquema mediante el cual la sociedad normalizada promueve la integración, estableciendo una relación de causalidad para explicar las limitaciones y carencias personales como determinantes y obstáculos al logro de una mejor calidad de vida, expresadas, en el caso de estudio, en la interpretada incapacidad parental del migrante. Es necesario subrayar que este proceso se ve afectado por las dificultades propias ocasionadas por la reunión de una familia que estuvo separada y que el reencuentro se genera en un espacio extraño que obliga a nuevos roles y estilos de comunicación (Marín, 2014).

No obstante, aparentemente se puede percibir un fenómeno contrario al estudiado en Estados Unidos por Huguet y Navarro (2006) y en Cataluña por Siguán (1998), en torno a que los estudiantes migrantes que llegan a ese país con menos años de escolaridad en su país de origen, tienen tiempo suficiente para alcanzar una relativa incorporación exitosa, en comparación con los que llegan a mayor edad a formar parte de cursos superiores. Para el caso estudiado en Arica, los estudiantes que vienen con escolaridad avanzada en su país de origen están en mejores condiciones, al verse favorecidos por ventajas curriculares, lo que les permite tener, con anticipación, el conocimiento de ciertos contenidos que los lleva a ser alumnos destacados en un contexto de acogida menos exigente, como el percibido en Chile. Este fenómeno genera a su vez, una respuesta de insatisfacción de parte de los padres migrantes, que evidencian que las deficiencias de la educación chilena perjudicaría un potencial retorno de sus hijos al país de origen (Bustos y Gairín, 2017). 
De acuerdo con lo aquí descrito, se reproduce lo que la literatura internacional señala en torno a que la mayoría de los estudiantes migrantes asiste a instituciones educativas donde los estándares de desempeño disminuyen la motivación de logro (SuárezOrozco, Suárez-Orozco y Todorova, 2008), lo que en el caso de Arica se representa mayoritariamente en las escuelas municipales.

Tal como reconocen Etxeberría y Elosegui (2010), a la mayor concentración del alumnado inmigrante en los centros públicos, en la región de estudio, se agrega la casi nula atención a las prácticas comunicativas y culturas de origen del alumnado inmigrante, así como la poca preparación del profesorado para enfrentar esta situación. Un número importante de profesores plantea como tarea pedagógica "integrar" al alumno "diferente" en el sistema educativo, interpretando las dificultades como un problema de un grupo particular y minoritario. De esta manera, la escuela es vista como la responsable de promover el patrón de "normalidad", estableciendo qué puede ser interpretado como buen alumno (Giovine y Martignoni, 2011). Esta percepción nace de la premisa que la diversidad es un problema, y como tal debiera ser negada, por lo que se asume que el rol de la escuela es funcionar como espacio de adaptación unilateral, promoviendo la aculturación y la renuncia de sus patrones culturales por parte del alumno "diferente" (Jiménez y Fardella, 2015).

Esta situación aparece tensionada con la aparición de la Ley de Inclusión Escolar (Ley n ${ }^{\circ} 20.845$, publicada el 8 de junio de 2015) y la Ley de No Discriminación (Ley n ${ }^{\circ} 20.609$, publicada el 24 de julio de 2013) que desnuda discursos que emergen de la preparación de los docentes, ya sea técnica o emocionalmente, para trabajar con un solo tipo de estudiante, el estudiante "normal" (Matus y Rojas, 2015).

Hannoun (1992) ya había propuesto modelos de gestión de la diversidad, caracterizados por diferentes actitudes ante este fenómeno. Su propuesta abarca desde las actitudes negativas hasta la diversidad, asociadas a la asimilación, pasando luego por la multiculturalidad (reconocimiento de las diferencias), llegando a la interculturalidad como valoración positiva de la diversidad cultural y de los intercambios propios nacidos en dicho contexto, 
generando síntesis deseadas de interacción social. De acuerdo con lo anterior, y refrendado por Essomba (2006), en la práctica, existiría una distinción entre multiculturalidad e interculturalidad, donde la primera se referiría a la diversidad cultural en un contexto, mientras que la segunda a las relaciones entre los componentes de dicha diversidad.

Para el caso en estudio, debemos señalar que la representación nacional se construye a la luz de la ficción de la homogeneidad, por lo que su predisposición a la interculturalidad es limitada y ajena. Este hecho lleva a que la construcción de un marco institucional para alcanzar mayores grados de inclusión de los migrantes en Chile, no solo requiere avances en el ámbito económico-laboral, sino que también en el área educacional-cultural, promoviendo una educación basada en la interculturalidad, capaz de marcar el camino hacia una mayor tolerancia. Sin embargo, es necesario relevar la alerta señalada por Diez (2004) en cuanto a que la educación no puede asumir por sí sola los desafíos que la interculturalidad supone, sin abordar realidades manifestadas en otros ámbitos de la vida social. Políticas que garanticen los derechos fundamentales y la igualdad de oportunidades para los migrantes, influirían positivamente en las actitudes hacia la interculturalidad.

\section{Conclusiones y nuevas propuestas}

En relación con los objetivos del estudio podemos concluir que Chile, al ser construido sobre la base del discurso de la homogeneidad nacional, muestra limitada predisposición a la interculturalidad. Principalmente esta situación se debe a la concepción dominante que privilegia la homogeneidad cultural como paradigma, lo que además deja entrever elementos de racismo de salida, traducidos en el convencimiento de que no hay que dar tratos especiales a nadie, en consecuencia con el concepto de igualdad e inclusión dominante en los establecimientos educativos. Las escuelas parten de la premisa de que es el estudiante quien debe adaptarse al sistema escolar, desde la mirada prevaleciente de la "integración". Esta "igualdad formal", considerada como tarea fundamental de los establecimientos, se reafirma a través de procedimientos normalizadores. Así, no es de 
extrañar que los mecanismos diseñados para apoyar al estudiante migrante se reduzcan al apoyo administrativo.

Por otro lado, la asociación del éxito de la adaptación con el rendimiento académico de los estudiantes tiende a que se justifique la ausencia de atención a sus particularidades, negando la diferencia y racionalizando el status quo. Aparentemente, los únicos mecanismos diseñados para apoyar al estudiante migrante y a su familia se refieren al apoyo administrativo y, en casos particulares, a cierto apoyo en el aula, el que nace como iniciativa personal de cada docente. Este último hecho da cuenta de cierta orfandad de las instituciones para enfrentar el desafío de la diversidad, haciendo urgente que la política pública expresada en la legislación que favorece la inclusión y reprime la discriminación, sea acompañada de iniciativas gubernamentales que permitan las condiciones para su cumplimiento.

Por otro lado, los padres migrantes se presentan agradecidos por las características del trato recibido. Sin embargo, este agradecimiento se relaciona mayormente con la posibilidad de haber recibido los mismos beneficios materiales (útiles escolares, desayuno, almuerzo, etc.) que los estudiantes nacionales y, en algunos casos, a la ausencia de castigos físicos como los que aparentemente eran comunes en algunos de los contextos educativos de origen. Esta misma percepción lleva a que los mismos padres y estudiantes migrantes perciban algunas conductas indeseables experimentadas en el contexto educativo de acogida, como "normales" o, mejor dicho, "esperables".

En cuanto a los tipos de estrategias investigadas, aparecen como carentes de iniciativas institucionales y solo presentes, como ya se dijo, en acciones esporádicas e individuales de algunos profesores. La razón esgrimida para esta situación alude a que no serían necesarias, en virtud de la motivación intrínseca de los estudiantes migrantes.

El tipo de estrategia con un incipiente nivel de implementación son aquellas de apoyo social donde las que aparecen como más evidentes se encuentran las de carácter actitudinal, pero como siempre, algunas veces como opción institucional y otras como iniciativa personal del docente. De todas maneras, las iniciativas 
coyunturales que asumen cierta formalidad se realizan aparentemente sin mayor conciencia de su rol en el contexto de una educación inclusiva.

Finalmente, es necesario señalar que si bien se alude en la investigación a un número de estrategias de control emocional, los profesores hacen responsables de estas a otros profesionales de apoyo a la docencia. En consecuencia, este tipo de estrategias asume una ejecución individual, caso a caso y divorciada del aula.

Respecto de las estrategias cognitivas, se trata de un elemento permanentemente presente en los discursos institucionales, en especial de los docentes, quienes señalan que ellas obedecen a iniciativas particulares de ellos mismos. En torno a ello, en algunos casos, se acepta la responsabilidad institucional en las labores de apoyo al estudiante migrante, sin embargo, siempre se percibe como un acto resultante de la acción del mismo, que lo haría, o no, merecedor de dicha acción. Así, la relación del establecimiento educacional con el estudiante migrante depende del desempeño individual de este y no de las condiciones generadas en el contexto de acogida. De esta manera, no hay opción para llevar a cabo una acción efectiva de cambio de situación del grupo más vulnerable.

Más específicamente, se pueden aportar desde el estudio algunas sugerencias de base. La primera releva la importancia de considerar las distintas trayectorias académicas de los estudiantes migrantes, adecuando la formación para aquellos grupos que ya conozcan los contenidos, a través de actividades que permitan mantener su desarrollo, facilitando así su incorporación social.

Es necesario, asimismo, prestar atención a los procesos de transición de estudiantes desde centros educativos rurales a urbanos, donde el número de estudiantes, el ambiente y la relación con los docentes genera desafíos que muchas veces no pueden superar fácilmente.

Por último, es necesario potenciar el rol que tienen los establecimientos educacionales como "puentes" entre las familias migrantes y su comunidad, ampliando el espacio de desarrollo de 
los estudiantes migrantes, evitando la conformación de trincheras familiares que conllevan a una doble marginación: externa, o nacida de las condiciones sociales, e interna, nacida de las propias familias migrantes que buscan protegerse mediante el aislamiento.

\section{Referencias}

Abajo, J. E. y Carrasco, S. (2004). Experiencias y trayectorias de éxito escolar de gitanas y gitanos en España. Recuperado de: https://www.gitanos. org/upload/04/70/1.5-EXP_Experiencias_y_trayectorias_de_exito_ escolar_de_gitanas_y_gitanos_en_Espana.pdf

Alvites, S. y Jiménez, A. (2011). Niños y niñas migrantes, desafío pendiente. Innovación educativa en escuela de Santiago de Chile. Synergies Chili, 7, 121-136. Recuperado de: https://gerflint.fr/Base/Chili7/alvites.pdf

Balibar, É. \& Wallerstein, I. (1998). Race, nation, clase. París: La Découverte.

Biblioteca del Congreso Nacional, (2015b). Reportes estadísticos Comunales. Recuperado de: http://reportescomunales.bcn.cl/2015/index.php/Arica

Biblioteca del Congreso Nacional (2015b).Resultados pruebas de selección universitaria (PSU) de la Comuna de Arica. Recuperado de: http:// reportescomunales.bcn.cl/2015/index.php/Arica.

Booth, T. y Ainscow, M. (2002). Índice de inclusión: desarrollando el aprendizaje y la participación en las escuelas. Santiago de Chile: Unesco-Orealc.

Bustos, R. y Gairín J. (2017). Expectativas académicas de estudiantes y padres migrantes: el caso de Arica, en la frontera de Chile y Perú. Revista Dilemas Contemporáneos: Educación, Política y Valores, 4(3). Recuperado de: http://files.dilemascontemporaneoseducacionpolitica yvalores.com/200003492-2cef52de79/17-5-23\%20Expectativas\%20 acad\%C3\%A9micas\%20de\%20estudiantes....pdf

Carrasco, S., Pàmies, J., y Bertrán M. (2009). Familias inmigrantes y escuela: desencuentros, estrategias y capital social. Revista Complutense de Educación, 20(1), 55-78. Recuperado de: http://revistas.ucm.es/index. php/RCED/article/viewFile/RCED0909120055A/15379

Centro de Estudios Mineduc. (2013). Informe sobre migrantes. Arica: Documento de trabajo Seremi de Arica y Parinacota. Departamento de planificación.

Diez, M. L. (2004). Reflexiones en torno a la interculturalidad. Cuadernos de Antropología Social, 19, 191-213. Recuperado de: http://www.scielo. org.ar/pdf/cas/n19/n19a12.pdf 
Dovigo, F. (2014). El tratamiento de la diversidad en las instituciones educativas. En J. Gairín (Coord.), Reflexiones y propuestas para la intervención $1^{\mathrm{a}}$ edición (pp. 87-118). España: Wolters Kluwer.

Etxeberría, F. y Elosegui, K. (2010). Integración del alumnado inmigrante: obstáculos y propuestas. Revista Española de Educación Comparada (16), 235-263. Recuperado de: http://www.uned.es/reec/pdfs/16-2010/11_ etxeberria.pdf

Essomba, M. A. (2006). Liderar escuelas interculturales e inclusivas. Equipos directivos y profesorado ante la diversidad cultural y la inmigración. Barcelona: Graó.

Figueroa, L. (2015). Construyendo "la identidad del excluido": Etnografía del aprendizaje situado de los niños en una escuela básica municipal de Chile. Estudios Pedagógicos, XLI, Nºspecial, 97-113. Recuperado de: http://mingaonline.uach.cl/pdf/estped/v4lnEspecial/art07.pdf

Gairín, J. (Coord.) (2014). Colectivos vulnerables en la universidad. Reflexiones y propuestas para la intervención. Madrid: Wolters Kluwer Educación. Recuperado de: https://www.dropbox.com/s/7jb4abuoqpyyloq/ ACCEDES\%201I_2014.pdf?dl=0

Gil, X. (1994). Categorización y complejidad en la investigación cualitativa. Revista de Investigación Educativa, 24(1), 535-537.

Giovine, R. y Martignoni, L. (2011). La escuela media bajo el mandato de la obligatoriedad. Cadernos CEDES, Campinas, 31(84), 175-194. https://doi.org/10.1590/s0101-32622011000200002

Glaser, B. \& Strauss, A. (1967). Discovery of grounded theory. Chicago: Aldine.

Gómez, J. (2005). Pautas y estrategias para entender y atender la diversidad en el aula. Pulso, 28, 199-214. Recuperado de: https://dialnet.unirioja. es/servlet/articulo? codigo $=1370936$

Hannoun, H. (1992). Els guettos de l'escola. Per una educació intercultural. Barcelona: EUMO Editorial.

Hernández, F., Martínez, S., y Montané, A. (2014). Micro etnografías y discontinuidad en una investigación sobre aprender a ser docente. En H. Cárcamo (Ed.), Making Of... Construcciones etnográficas de la educación (pp. 65-71). Recuperado de: http://e-spacio.uned.es/fez/ eserv/bibliuned:500383-IIICongresoEtnografia-1005/Documento.pdf

Hombrados, I. y Castro, M. (2013). Apoyo social, clima social y percepción de conflictos en un contexto educativo intercultural. Anales de Psicología, 1(29), 108-122. http://dx.doi.org/10.6018/analesps.29.1.123311

Huguet, A. y Navarro, J.L. (2006). Inmigración y resultados escolares: lo que dice la investigación. Cultura y Educación, 18 (2), 117-126. 
Instituto Nacional de Estadísticas, INE. (2012). Censo 2012: Resultados XVIII Censo de Población. Chile: Biblioteca del Congreso Nacional. Recuperado de: http://indigenousnews.org/wp-content/ uploads/2013/04/resultados_censo_2012_poblacion_vivienda_ tomoslyII.pdf

Jackson, P. (2001). La vida en las aulas. Madrid: Morata.

Jiménez, F. y Fardella, C. (2015). Diversidad y rol de la escuela. Discursos del profesorado en contextos educativos multiculturales en clave migratoria. Revista Mexicana de Investigación Educativa RMIE, 20(65), 419-441. Recuperado de: http://www.redalyc.org/ pdf/140/14035408005.pdf

Joiko, S. y Vásquez, A. (2016). Acceso y elección escolar de familias migrantes en Chile: No tuve problemas porque la escuela es abierta, porque acepta muchas nacionalidades. Calidad en la Educación, 45, 132-173. https://doi.org/10.4067/s0718-45652016000200005

Lanza, D. y Sánchez, V. (2013). Estrategias de aprendizaje en educación secundaria: un estudio comparativo sobre su uso entre alumnos españoles e inmigrantes. European Journal of Investigation in Health, Psychology and Education, 3(3), 227-236. Recuperado de: https:// dialnet.unirioja.es/descarga/articulo/4916982.pdf

Ley n 20.370 Establece Ley General de Educación. Diario Oficial de la República de Chile, Santiago, Chile, 25 de mayo de 2016. Recuperado de: http://www.anfitrion.cl/ley/20370.html

Lube, M., Penna, C., Vicuña, J., y Pérez, C. (2015). Claves conceptuales e históricas para comprender la frontera norte de Chile y la migración en Arica y Parinacota. En J. T. Vicuña y T. E. Rojas (Eds.), Migración internacional en Arica y Parinacota (pp. 19-36). Santiago: Universidad Alberto Hurtado.

Marín, J. (2014). Hijos/as de inmigrantes en el norte de Chile: Hacia la construcción de una escuela intercultural. Santiago de Chile: Haz tu tesis en cultura. Recuperado de: http://www.observatoriocultural.gob.cl/wpcontent/ uploads/2015/05/tesis_jair_marin.pdf

Matus, C. y Rojas, C. (2015). Normalidad y diferencia en nuestras escuelas: a propósito de la Ley de Inclusión Escolar. Docencia, 56, 47-56. Recuperado de: http://www.revistadocencia.cl/new/wp-content/ pdf/20150826143709.pdf

Miles, M. \& Huberman, A. M. (1994). Data management and analysis methods. En N. K. Denzin e Y. S. Lincoln (Eds.), Handbook of qualitative research (pp. 39-43). London: Sage Publication. 
Mondaca, C. y Gajardo, Y. (2013). La educación intercultural bilingüe en la región de Arica y Parinacota, 1980-2010. Diálogo Andino, 42, 69-87. http://dx.doi.org/10.4067/S0719-26812013000200007

Morales, V. y Molina Prieto, C. A. (2003). Reasentamiento involuntario: integración y civilización. Revista Bitácora Urbano Territorial, 1(7), 1925. Recuperado de: http://www.redalyc.org/articulo.oa?id=74810704

Moscoso, M. (2014). Acerca del enfoque (auto) biográfico aplicado a la investigación con niños y niñas. En H. Cárcamo (Ed.), Making of... Construcciones etnográficas de la educación (pp. 93-98). Recuperado de: http://e-spacio.uned.es/fez/eserv/bibliuned:500383IIICongresoEtnografia-1005/Documento.pdf

Oficina Internacional para las Migraciones, OIM. (2013). Informe sobre las migraciones en el mundo. Ciudad: Alcoy. Recuperado de: http:// publications.iom.int/bookstore/free/WMR2013_SP.pdf

Pàmies, J. (2011). Éxito académico, inmigración y ciudadanía. Condiciones y posibilidades entre jóvenes de origen marroquí en Cataluña. En F. J. García Castaño y N. Kressova (Coords.), Actas del I Congreso Internacional sobre Migraciones en Andalucía (pp. 443-448). Granada: Instituto de Migraciones. Recuperado de: http://migraciones.ugr.es/ congreso2011/libroacta/Mesa5/Mesa5.pdf

Pizarro, C. (2011). Introducción. En C. Pizarro (Ed.), Migraciones internacionales contemporáneas. Estudios para el debate (pp. 5-17). Buenos Aires: Ciccus.

Pidgeon, N. \& Henwood, K. (1997). Grounded theory: Practical implementation. En J. Richardson (Comp.), Handbook of qualitative research methods for Psychology and the Social Sciences (pp. 86-101). Leicester: BPS Books.

Poblete, R. (2006). Educación intercultural: teorías, políticas y prácticas. La migración peruana en el Chile de hoy. Nuevos escenarios y desafíos para la integración (Tesis doctoral, Universidad Autónoma de Barcelona, España). Recuperado de: http://www.tesisenred.net/bitstream/ handle/10803/5519/rpmldel.pdf?sequence=1

Poveda, D. (2003). Saberes sociolingüísticos en una clase multicultural. En D. Poveda (Comp.), Entre la diferencia y el conflicto. Miradas etnográficas a la diversidad cultural en educación (pp. 67-98). Cuenca: Ediciones de la Universidad de Castilla-La Mancha.

Riedemann, A. y Stefoni, C. (2015). Sobre el racismo, su negación, y las consecuencias para una educación anti-racista en la enseñanza secundaria chilena. Polis Revista Latinoamericana, 42. Recuperado de: http://polis.revues.org/11327 
Sánchez, H. y Gil, I. (2015). Análisis interseccional y enfoque intercultural en el estudio de la ciudadanía y la participación. Consideraciones epistemológicas. Diálogo Andino, 47, 143-149. https://doi.org/10.4067/s0719-26812015000200015

Sauquillo, J. (1987). Poder político y sociedad normalizada en Michel Foucault. Revista de Estudios Políticos (Nueva Época), 56, 181-203. Recuperado de: https://dialnet.unirioja.es/descarga/articulo/26930.pdf

Siguan, M. (1998). La escuela y los inmigrantes. Barcelona: Paidós

Stefoni, C. (2003). Inmigración peruana en Chile: una oportunidad a la integración. Santiago de Chile: Editorial Universitaria.

Suárez-Orozco, M; Suárez-Orozco, C y Todorova, I. (2008). Learning a new land: immigrant students in American Society. Cambridge: Harvard University Press

Vázquez, A. y Martínez, I. (1996). La socialización en la escuela. Una perspectiva etnográfica. Barcelona: Paidós.

Vera, R. (2009). Análisis psicosocial a partir de los enfoques teóricos sobre el comportamiento colectivo y los movimientos sociales. Recuperado de: http://medicalia.ning.com/profiles/blog/list?user=0t829n4x9bgbp

Vicuña, J. y Rojas, T. (2015). Características sociodemográficas de la migración en Arica y Parinacota. En J. Vicuña y T. Rojas (Eds.), Migración internacional en Arica y Parinacota: panoramas y tendencias de una región fronteriza (pp. 87-104). Santiago: Ediciones Universidad Alberto Hurtado.

Zanetti, G. (2015). Reflexiones sobre la igualdad a la luz de la Teoría Crítica de la Raza (Critical Race Theory). Derechos y Libertades, 33, 47-65. Recuperado de: http://e-archivo.uc3m.es/bitstream/ handle/10016/24123/DyL-2015-33-zaneti.pdf?sequence=1

Recibido: 17/09/2016

Aceptado: 30/05/2017 